\title{
Infección por Rhodococcus equi en pacientes con SIDA. Análisis retrospectivo de 13 pacientes en Argentina
}

\author{
Marcelo Corti, Rubén Solari, Luis De Carolis, Omar Palmieri, Raquel Rollet y Haroun N. Shah
}

\author{
Rhodococcus equi infection in AIDS patients. \\ Retrospective analysis of 13 patients in Argentina
}

Introduction: Rhodococcus equi is a gram positive coccoid rod that causes pulmonary infections in immunosuppressed patients. Methods: We retrospectively analyzed epidemiological, clinical, microbiological, radiological, and immunological features as well as the outcomes of 13 AIDS patients with $R$. equi infection. Results: Between January 1994 and December 2012, 13 patients attending the AIDS department of the Infectious Diseases reference hospital in Buenos Aires were diagnosed with $R$. equi infection. All were men, the median age was 27 years. At the time of diagnosis, the median of CD4+ T cell counts was 11 cells/ $\mu 1$. Twelve patients presented pulmonary disease with isolation of the microorganism from sputum or bronchoalveolar lavage; in the other patient the diagnosis was postmortem with positive culture of cerebrospinal fluid. The most frequent clinical manifestations were fever, haemoptysis, and weight loss. The predominant radiological finding was lobe consolidation with cavitation. Nine patients died after a median survival of 5.5 months. In all of them, cultures persisted positive until the last admission. The other 4 patients did continue clinical follow-ups. Conclusion: The insidious course of $R$. equi disease and the difficulties in the isolation of the microorganism contribute to the delay in the diagnosis and to the high mortality rate of this opportunistic infection.

Key words: Rhodococcus equi, pneumonia, HIV, AIDS.

Palabras clave: Rhodococcus equi, neumonía, VIH, SIDA.

\section{Introducción}

$R$ hodococcus equi es un cocobacilo grampositivo pleomórfico, parcial o débilmente ácido resistente, cuyo nombre se debe a los característicos cambios cíclicos de su forma cocobacilar: "rod or coccus". Desarrolla en los medios de cultivo dando colonias pigmentadas de un color rosa salmón característico'.

Fue identificado por primera vez en 1923 por Magnusson en equinos ${ }^{2}$ y el primer caso de enfermedad en humanos fue descrito por Golub en $1967^{3}$. La enfermedad por $R$. equi es una complicación poco frecuente en los pacientes con grave deterioro de la inmunidad celular, y se produce en especial, en aquéllos con SIDA. La especie $R$. equi constituye un taxón heterogéneo que podría incluir varias especies, y cuya diferenciación de otros actinomicetales e identificación puede requerir estudios moleculares ${ }^{4}$. El compromiso pulmonar es la forma más frecuente de presentación clínica de la enfermedad por $R$. equi en pacientes con SIDA.

En este trabajo se presenta un análisis retrospectivo de 13 pacientes con enfermedad por VIH/SIDA avanzada que desarrollaron infecciones por R. equi.

\section{Métodos}

Se trata de un estudio retrospectivo, descriptivo, en el que se analizaron las características epidemiológicas, clínicas, microbiológicas, imagenológicas, inmunológicas y la evolución de 13 pacientes con diagnóstico de enfermedad VIH/SIDA que egresaron del Hospital de Infecciosas "F. J. Muñiz" con diagnóstico de infección por $R$. equi entre enero de 1994 y diciembre de 2012. La búsqueda se efectuó a partir de la revisión de los diagnósticos de egreso durante el período de estudio y de los libros de registro de la Unidad de Bacteriología del Hospital. Rhodococcus equi fue aislado de muestras respiratorias, de sangre y líquido cefalorraquídeo (LCR).

Las muestras de esputo fueron extendidas sobre portaobjetos, coloreadas con las técnicas de Gram, ZiehlNeelsen y Kinjoun y examinadas al microscopio (100X). Las mismas se sembraron en placas de agar sangre de cordero y agar chocolate mediante el método de descarga en cuatro estrías. Las muestras obtenidas por lavado bronco-alveolar (LBA) fueron sembradas con técnica cuantitativa en placas de agar sangre y agar chocolate y los sedimentos se extendieron para realizar los exámenes

\author{
Universidad de Buenos Aires, \\ Bs. Aires, Argentina. \\ Facultad de Medicina \\ Departamento de Medicina, \\ Orientación Enfermedades \\ Infecciosas, (MC, RS, LDC, OP) \\ Hospital de Enfermedades \\ Infecciosas F. J. Muñiz, Buenos \\ Aires, Argentina. \\ Unidad de Bacteriología (RR). \\ University of London, England \\ Health Public Protection Agency \\ (HNS).
}

Los autores declaran no tener conflictos de interés.

Recibido: 28 de diciembre de 2013 Aceptado: 30 de junio de 2014

Correspondencia a:

Marcelo Corti

marcelocorti@fibertel.com.ar 
microscópicos con las mismas coloraciones mencionadas para los esputos. Los medios de cultivo se incubaron a $35^{\circ} \mathrm{C}$ por 5 a 7 días y se observaron a diario para la detección de colonias de bacterias con potencial neumopatógeno y que, en el caso de las muestras obtenidas por LBA, superaran la concentración de $10.000 \mathrm{ufc} / \mathrm{mL}$. Las muestras de sangre fueron obtenidas por dos punciones y sembradas en frascos para hemocultivos de Biomérieux (Francia) e incubadas a $35^{\circ} \mathrm{C}$ durante 7 días. Los cultivos positivos fueron subcultivados en placas de agar sangre de cordero y agar chocolate y extendidos y coloreados con la tinción de Gram. El procesamiento inicial de las muestras de LCR consistió en el cultivo en medios sólidos de agar sangre de cordero, agar chocolate y caldo de tioglicolato y se incubaron a $35^{\circ} \mathrm{C}$ por un tiempo máximo de 7 días. Ante el desarrollo de colonias mucoides, de color rosasalmón se procedió a su identificación mediante el examen microscópico de extendidos en fresco coloreados con la tinción de Gram, Ziehl- Neelsen y Kinjoun posteriores a la centrifugación. Para comprobar la ausencia de micelios aéreos se realizaron microcultivos en agar-agua. Se realizó una batería de pruebas bioquímicas incluyendo la prueba de CAMP con la cepa Staphylococcus aureus ATCC 25923, observándose en todos los casos la típica punta de flecha por exaltación de la hemólisis de $S$. aureus. Se evaluó y observó la producción de catalasa y ureasa y la hidrólisis de adenina, xantina, hipoxantina y tirosina. Las pruebas de oxidación/fermentación fueron realizadas en tubos utilizando el medio de Gordon con el agregado del hidrato de carbono correspondiente en una concentración final de 1\%. En el año 2001, los aislados procedentes de los materiales clínicos de 10 de los pacientes fueron analizados en el Molecular Identification Service Unit, Health Protection Agency, Center for Infections, London, U.K., actualmente Public Health England (PHE), donde fueron estudiados mediante cromatografía gaseosa, espectrometría de masa y secuenciación de ARNr 16S. Este último método fue utilizado como referencia.

Para el análisis por cromatografía gaseosa, los aislados se cultivaron en caldo de tripticasa-soja a $30^{\circ} \mathrm{C}$ durante $48 \mathrm{~h}$; los ácidos grasos de cadena larga fueron extraídos y analizados (MIDI Sherlock, Newark, NJ, USA). Mediante espectrometría de masa MALDITOF-MS (matrix-assisted laser desorption/ionisation time-of-flight mass spectrometry), se analizaron las moléculas superficiales de estos 10 aislados y los perfiles obtenidos fueron alineados con los de la base de datos existentes. Este método también permitió identificar a todos los aislados estudiados como pertenecientes a $R$. equi.

Mediante la utilización de partidores universales para ADNr 16S se amplificó y posteriormente se secuenció y se evaluó (GenBank) el fragmento amplificado. Las pruebas bioquímicas y moleculares permitieron identificar a los microorganismos caracterizados como $R$. equi.
En siete aislados se efectuaron pruebas de sensibilidad a antimicrobianos, determinándose la concentración inhibitoria mínima (CIM) por el método de microdilución en caldo, disponible en nuestro medio, (Microscan paneles para Staphylococcus) o E-test (AB Biodisk, Solna, Sweden) [National Committee for Clinical Laboratory Standard. Susceptibility Testing for Mycobacteria, Nocardiae and other aerobic Actinomycetes. Wayne, Pennsylvania, U.S.A. 2003], que no establece puntos de corte para la interpretación de la sensibilidad.. Para los siete aislados se probaron los siguientes antimicrobianos: ciprofloxacina, imipenem, rifampicina y vancomicina; claritromicina se probó sólo para cuatro.

Finalmente, se evaluó la respuesta clínica y microbiológica al tratamiento y la supervivencia de los pacientes.

\section{Resultados}

En el período de estudio, 0,053\% del total de pacientes con SIDA egresó con diagnóstico de infección por $R$. equi. La totalidad de la muestra estuvo integrada por varones, con una mediana de edad de 27 años (rango: 23 a 37 años). En 11 pacientes (84,6\%), la enfermedad por $R$. equi fue la primera manifestación de la infección por el retrovirus. $\mathrm{La}$ serología para el virus de la hepatitis C (VHC) se realizó en 10 pacientes con 9 resultados positivos. La mediana del recuento de linfocitos T CD4 + en el momento del diagnóstico de la infección por $R$. equi fue de 11 céls/ $\mu l$ (rango: 0 a 170 céls $/ \mu 1$ ), y sólo en dos pacientes este valor fue mayor a 50 céls $/ \mu 1$. Ninguno de ellos recibía terapia anti-retroviral de gran actividad (TARGA) al momento del diagnóstico. En cuanto a los antecedentes epidemiológicos, sólo un paciente había tenido contacto con caballos, debido a su actividad laboral.

Las manifestaciones clínicas predominantes incluyeron fiebre en $100 \%$ de los casos, pérdida de peso en $46 \%$, hemoptisis en $46 \%$, tos con expectoración mucopurulenta en $38 \%$ y tos con expectoración hemoptoica en $30 \%$.

Las alteraciones más frecuentes de los parámetros bioquímicos fueron la velocidad de eritrosedimentación acelerada en $100 \%$ de los pacientes y anemia en $62 \%$ de los casos. En tres pacientes se comprobó elevación significativa de los valores de la láctico deshidrogenada (LDH).

La radiografía de tórax mostró anormalidades en 12 pacientes $(92,3 \%)$; en 10 casos $(76,9 \%)$ se apreciaron imágenes de consolidación lobar con cavitación, sin predominio de algún área pulmonar. Dos pacientes presentaron infiltrados heterogéneos bilaterales y en uno, la radiografía de tórax resultó normal. La tomografía axial computarizada (TAC) de tórax se efectuó en tres pacientes; en dos de ellos las imágenes fueron de consolidación lobar, con cavidades que ya se habían observado en las radiografías simples; en el restante se comprobó cavitación no evidenciada en las radiografías previas. 
La identificación presuntiva de $R$. equi se realizó mediante pruebas bioquímicas convencionales en aislados obtenidos de 11 muestras de esputo, cinco de LBA, dos de hemocultivos y uno de LCR. En aislados obtenidos de 10 pacientes, esta identificación inicial se confirmó por secuenciación del gen ARNr 16S. El perfil de ácidos grasos correspondió a $R$. equi.

Los rangos de CIMs obtenidos en $\mu \mathrm{g} / \mathrm{ml}$ fueron: ciprofloxacina: $\leq 1-2$, claritromicina: $\leq 0,06-0,12$; imipenem: $\leq 4$, rifampicina: $\leq 1$ y vancomicina: $\leq 1$. En tres pacientes se comprobaron co-infecciones respiratorias con aislamiento simultáneo de Streptococcus pneumoniae, Haemophilus influenzae y Pneumocystis jiroveci. Para los aislados presentados, la CIM de los cinco antibacterianos probados fue inferior a las concentraciones séricas que estos agentes alcanzan habitualmente.

El tratamiento de esta complicación, que comprendió dos semanas iniciales de administración intravenosa para luego pasar a la vía oral, incluyó en 11 casos combinaciones entre los siguientes antimicrobianos: vancomicina, rifampicina, claritromicina y ciprofloxacina. Un paciente recibió monoterapia con imipenem.

La mediana de seguimiento fue de seis meses; nueve pacientes fallecieron durante las internaciones por la infección por $R$. equi con una mediana de supervivencia de 5,5 meses. Durante el lapso de seguimiento, los cultivos de esputo permanecieron positivos en todos estos pacientes. Los cuatro restantes se perdieron para el seguimiento. En un paciente la autopsia permitió identificar a $R$. equi en los exámenes bacteriológicos de las muestras pulmonares.

\section{Discusión}

Rhodococcus equi es una bacteria grampositiva parcial o débilmente ácido resistente. El aislamiento de bacterias corineformes ácido resistentes, de colonias irregulares, muchas veces mucosas, extendidas sobre la línea de siembra y que, a través del tiempo, adquieren un color rosa salmón de intensidad variable, debe ser evaluado en el contexto clínico epidemiológico de los pacientes. Sin embargo, también se ha descrito el aislamiento de colonias no pigmentadas. Se desconoce la proporción de estas últimas y su valor clínico en seres humanos. Por cierto, estas cepas no pigmentadas pueden subestimarse si la búsqueda e identificación de $R$. equi se basa exclusivamente en el pigmento de las colonias. Aun cuando su identificación puede presentar dificultades, su presencia en los materiales normalmente estériles no representa problema alguno de interpretación; pero en los materiales habitualmente contaminados, como las muestras de esputo, pueden confundirse con bacterias difteroides de la microbiota habitual y ser considerados como contaminantes con la consiguiente subestimación y retraso en el diagnóstico.
Por este motivo, debe prestarse especial atención a los exámenes microscópicos y a los cultivos de los esputos de pacientes en riesgo de contraer esta infección. En los laboratorios clínicos, la identificación de la especie $R$. equi se basa en la morfología microscópica, el aspecto de las colonias y en pruebas bioquímicas y metabólicas; la clasificación taxonómica se basa en métodos de biología molecular como la composición de ácidos grasos de la pared, ARNr S16, etc.

Se ha señalado que el cultivo de esputo representa el método con más posibilidades para el diagnóstico ${ }^{5}$. En la serie que se analiza, en 11 de 12 pacientes con neumonía (91\%) fue posible identificar al agente causal a partir de las muestras de esputo. En nuestra experiencia, todos los aislados de $R$. equi procedentes de muestras de esputo de pacientes infectados con VIH resultaron ser significativos. En este sentido, podemos decir que el aislamiento de colonias con las características señaladas y procedentes de materiales clínicos de pacientes con un marcado deterioro de la inmunidad celular, debe llevar a la realización de pruebas para demostrar la morfología cíclica y ácido resistencia, así como la prueba de CAMP y la de utilización de adenina. Con estas pruebas mínimas y en el contexto clínico señalado, se puede identificar $R$. equi en forma presuntiva. Su confirmación requiere la realización de pruebas moleculares como la secuenciación genética. De esta manera, podemos afirmar que, al igual que en series de casos publicadas por otros autores, el cultivo de esputo ha sido para nosotros una herramienta diagnóstica sumamente válida ${ }^{1,3,6}$.

El LBA y los hemocultivos son también materiales útiles para la identificación del microorganismo, y por cierto, se contaminan con menos frecuencia. Los hemocultivos suelen ser positivos hasta en $50 \%$ de los pacientes con SIDA $^{7,8}$. Sin embargo, para esta presentación, sólo hemos evaluado los hemocultivos positivos sin corroborar todas las solicitudes ni otros resultados; por lo cual no podemos analizar ni concluir sobre la sensibilidad de los hemocultivos para el diagnóstico de esta infección. No obstante, se reconoce el valor significativo de los aislados de $R$. equi de estas muestras así como de los materiales de LBA cuando se respetan los criterios habituales de celularidad y recuento mayor de $10^{4} \mathrm{ufc} / \mathrm{ml}$ que habitualmente se utilizan para su interpretación.

Si bien se ha postulado que el ser humano se infecta por contacto con animales, son numerosos los casos de infección por $R$. equi en los que dicho contacto no se ha demostrado. En esta muestra, sólo uno de los trece pacientes refirió haber tenido contacto previo con caballos. Por lo tanto, en nuestra opinión, este antecedente epidemiológico no parece ser relevante ${ }^{4,9}$.

La mayoría de los casos de enfermedad por $R$. equi se ha descrito en pacientes con SIDA. Las dos terceras partes de las infecciones por este patógeno se dan en esta pobla- 
ción ${ }^{10}$, y sólo algunos casos aislados se han presentado en pacientes bajo corticoterapia prolongada, tratamiento con otros agentes inmunosupresores, receptores de trasplantes, alcohólicos y pacientes con neoplasias ${ }^{2,10,11}$.

En la población de pacientes con SIDA, esta complicación se presenta en estadios avanzados de la inmunodeficiencia. Torres-Tortosa y cols. ${ }^{8}$, publicaron 67 casos de enfermedad por $R$. equi en pacientes con serología reactiva para VIH, en los que la media de linfocitos T CD4 + fue de 35 céls/ $\mu 1$. En 11 pacientes de la serie que se presenta, la infección por $R$. equi fue la primera manifestación de la inmunodeficiencia asociada con el retrovirus, la mayoría de ellos presentaba infección concomitante con VHC y la mediana del recuento de LT CD4 + al momento del diagnóstico fue de 11 céls $/ \mu 1$.

Rhodococcus equi se disemina por vía hemática y el pulmón es el parénquima involucrado con mayor frecuencia ${ }^{8}$. En dos series de pacientes, el pulmón se vio involucrado en más de $90 \%$ de los pacientes analizados ${ }^{5}$. Sin embargo, en una revisión de casos que afectaron a pacientes sin infección por VIH, el compromiso pulmonar alcanzó a 27 de 54 (50\%) pacientes estudiados ${ }^{5,11}$. Por lo tanto, puede considerarse que la forma de presentación con síntomas respiratorios es la más frecuente en pacientes con SIDA, y $R$. equi debe incluirse en el diagnóstico diferencial de los patógenos que pueden provocar complicaciones pulmonares en estos enfermos ${ }^{5}$.

En relación con las lesiones observadas en las imágenes del tórax, el compromiso lobar y la cavitación son los hallazgos más comunes referidos en la literatura científica $^{8}$, que también se pudieron comprobar en la serie que se presenta. La cavitación se observa en dos tercios de los casos, por lo que $R$. equi debe incluirse en el diagnóstico diferencial de las neumonías cavitadas de los pacientes con SIDA ${ }^{3,5,6,8,11}$. Meng ZH y cols. ${ }^{12}$, evaluaron a 15 pacientes con SIDA e infección por $R$. equi de los cuales 14 se presentaron como neumonías lobares o abscesos de pulmón. En la serie que motiva el presente trabajo, la consolidación con cavitación se observó en 10 de 13 casos $(76,9 \%)$.

Los datos de susceptibilidad in vitro existentes en la literatura médica internacional dan cuenta, mayormente, de la sensibilidad de cepas aisladas del medio ambiente y de animales y, aquellas que consignan aislados humanos clínicamente significativos, presentan un bajo número de cepas. En este aspecto es destacable el trabajo publicado por McNeil y Brown en $1992^{13}$, quienes estudiaron una serie de 98 aislados humanos mediante el método de microdilución. El patrón de sensibilidad a los antimicrobianos de esta bacteria puede presentar variaciones geográficas $^{13}$. En esta serie, este dato estuvo disponible en siete casos y no mostró diferencias con respecto a otras en la sensibilidad a los antimicrobianos de uso habitual para el tratamiento de las infecciones por $R$. equi $i^{13,14}$.
El documento NCCLS (CLSI) vigente a la fecha de los aislados de esta serie no establecía puntos de corte para la interpretación de la sensibilidad a los antimicrobianos. De todas formas, si ahora aplicáramos los criterios de interpretación para $S$. aureus dados en el documento M100 del CLSI [Clinical and Laboratory Standars Institute. Susceptibility Testing of Mycobacteria, Nocardiae and other Anaerobic Actinomycetes; Approved Standard. Second Edition. M24A2. Vol $31 N^{\circ}$ 5] tal como lo indican las normativas actuales, los aislados de los pacientes incluidos en esta serie serían interpretados como sensibles a los antimicrobianos ensayados. No se encontraron cepas resistentes a imipenem; no obstante, se ha demostrado la emergencia de resistencia durante el tratamiento con este antibacteriano ${ }^{9}$. En concordancia con otras publicaciones $^{4,13,14}$, todos los aislados presentados en esta serie fueron inhibidos por concentraciones de vancomicina $\leq 2 \mu \mathrm{g} / \mathrm{ml}$. Como excepciones se han presentado dos aislados de la serie de McNeil y Brown ${ }^{13}$ y tres de siete cepas identificadas en Taiwan ${ }^{14}$ que resultaron resistentes a este antibacteriano. Además, Guardabassi y cols. ${ }^{15}$, han detectado la presencia de genes vanA y vanB (homólogos a los hallados en enterococos resistentes a los glicopéptidos) pero en cepas de Rhodococcus aisladas del suelo. Topino $\mathrm{S}$ y cols. ${ }^{16}$, en una revisión de 272 casos de rodococosis en pacientes con infección por VIH, señalan que las cepas de $R$. equi identificadas en esta serie mostraron una baja sensibilidad a ceftriaxona, amoxicilina/ácido clavulánico y cotrimoxazol.

Además, ya que $R$. equi es una bacteria que puede vivir dentro de los macrófagos, es importante conocer la sensibilidad a antimicrobianos con actividad intracelular como son los macrólidos, rifampicina, tetraciclinas y quinolonas. En el trabajo que se presenta, rifampicina, claritromicina y ciprofloxacina mostraron actividad a las concentraciones alcanzables en suero. En un estudio llevado a cabo por Jacks y cols. ${ }^{17}$, con 64 aislados procedentes de potrillos, eritromicina, claritromicina y azitromicina fueron agentes muy activos al igual que rifampicina, gentamicina e imipenem, y sus CIMs fueron inferiores a las alcanzadas en las secreciones respiratorias y en el tejido pulmonar equino. Eritromicina y rifampicina han sido muy utilizadas para el tratamiento de la neumonía por $R$. equi en potrillos y posteriormente en seres humanos. Actualmente, se propone que uno de estos dos agentes forme parte de todas las etapas del tratamiento. La particular evolución que experimentan los pacientes con SIDA y neumonía por $R$. equi, obliga a implementar esquemas terapéuticos basados en antimicrobianos con actividad bactericida intracelular, administrados inicialmente por vía intravenosa y durante un tiempo prolongado. En este sentido, la asociación más recomendada para el comienzo del tratamiento por vía intravenosa es vancomicina más imipenem o rifampicina. Los fármacos lipofílicos con 
buena penetración intracelular también resultan útiles, como las combinaciones de claritromicina o ciprofloxacina más rifampicina ${ }^{13,14,17}$. El drenaje de los abscesos y la resección quirúrgica de los segmentos o lóbulos pulmonares comprometidos debe limitarse a aquellos pacientes que no responden al tratamiento médico ${ }^{8,18,19}$. La duración del tratamiento de estos pacientes no está bien establecida; sin embargo, al igual que ocurre con otras infecciones oportunistas en pacientes con SIDA, es probable que luego de dos semanas iniciales de terapia intravenosa pueda pasarse a la vía oral y mantener la antibioticoterapia hasta la negativización de los estudios microbiológicos y la reconstitución inmunológica asociada con la TARGA.

La introducción precoz de la TARGA puede modificar la evolución y pronóstico de esta complicación. En la serie de Torres-Tortosa y cols. ${ }^{8}$, la ausencia de TARGA fue la única variable asociada con la mortalidad por $R$. equi, probablemente en relación con el grave deterioro inmunológico que muestran la mayoría de los pacientes en el momento del diagnóstico.

En el conocimiento de los autores, esta es la serie más amplia de pacientes con SIDA e infección por $R$. equi evaluada en Argentina. Si bien la muestra es pequeña y no permite sacar conclusiones definitivas, los resultados obtenidos aportan datos de interés, especialmente si se tiene en cuenta la baja incidencia de esta infección oportunista. La infección por $R$. equi es una complicación grave en los pacientes con SIDA, que mostró en nuestra serie una alta tasa de letalidad, escasa respuesta microbiana a la terapia y persistencia del patógeno durante varios meses luego del episodio agudo.

En conclusión, la infección por $R$. equi es una complicación infrecuente en pacientes con SIDA, pero con una elevada tasa de letalidad. La principal localización de la infección por $R$. equi en esta serie fue la pulmonar y su presentación clínica incluyó la fiebre, pérdida de peso, tos con expectoración mucopurulenta y/o hemoptoica y la presencia de consolidación con cavitación como hallazgo radiológico más frecuente. El diagnóstico precoz, el tratamiento antimicrobiano combinado y prolongado y el inicio de la TARGA en forma temprana pueden mejorar la evolución y el pronóstico de estos pacientes.

\section{Resumen}

Introducción: Rhodococcus equi es un cocobacilo grampositivo que provoca compromiso pulmonar en pacientes inmunodeprimidos. Métodos: En el presente trabajo se analizaron de manera retrospectiva los hallazgos epidemiológicos, clínicos, microbiológicos, imagenológicos, inmunológicos y la evolución de 13 pacientes con SIDA y enfermedad por R. equi. Resultados: Entre enero de 1994 y diciembre de 2012, 13 pacientes internados en la División de VIH/SIDA del hospital de referencia para Enfermedades Infecciosas de la ciudad de Buenos Aires egresaron con diagnóstico de enfermedad por $R$. equi. Todos eran varones y la mediana de edad fue 27 años. La mediana de linfocitos T CD4+ fue de 11 céls/ $\mu 1$. Doce pacientes presentaron enfermedad pulmonar con aislamiento del microorganismo del esputo o del lavado bronco-alveolar; en el restante se recibió post mortem el cultivo positivo de líquido cefalorraquídeo. Las manifestaciones clínicas más frecuentes fueron fiebre, hemoptisis y pérdida de peso. La imagen radiológica predominante fue la consolidación con cavitación. Nueve pacientes fallecieron, con una mediana de supervivencia de 5,5 meses. En todos ellos el cultivo persistió positivo hasta la última internación. Los cuatro restantes abandonaron los controles y no pudieron ser evaluados en el tiempo. Conclusión: El curso insidioso de la enfermedad por $R$. equi y las dificultades en la identificación del microorganismo, contribuyen al retardo en el diagnóstico y a la elevada mortalidad de esta infección oportunista en esta población de pacientes.

\section{Referencias bibliográficas}

1.- Verville T D, Huycke M M, Greenfield R A, Fine D P, Kuhls T L, Slater L N. Rhodococcus equi infections of humans. Medicine 1994; 73. 119-32.

2.- Rabagliati R, Morales A, Baudrand R, Jorquera J, Oddó D, García P, et al. Neumonía cavitada por Rhodococcus equi en paciente inmunocomprometido no infectado por virus de inmunodeficiencia humana. Caso clínico y revisión. Rev Chilena Infectol 2005; 22 : 155-60.

3.- Golub B, Falk G, Spink W W. Lung abscesses due to Corynebacterium equi: report of the first human infection. Ann Intern Med 1967;
66: 1174-7.

4.- McMinn E J, Alderson G, Dodson H I, Goodfellow M, Ward A C. Genomic and phenomic differentiation of Rhodococcus equi and related strains. Antonie Van Leeuwenhoek 2000; 78: 331-40.

5.- Donisi A, Suardi M G, Casari S, Longo M, Cadeo G P, Carosi G. Rhodococcus equi infection in HIV-infected patients. AIDS 1996; 10: 359-62.

6.- Scott M A, Graham B S, Verrall R, Dixon R, Schaffner W, Tham K T. Rhodococcus equi an increasingly recognized opportunistic pathogen. Report of 12 cases and review of 65 cases in the literature. Am J Clin Pathol 1995; 103 : $649-55$.
7.- Prescott J F. Rhodococcus equi: an animal and human pathogen. Clin Microbiol Rev 1991; 4: 20-34

8.- Torres-Tortosa M, Arrizabalaga J, Villanueva J L, Gálvez J, Leyes M, Valencia E, et al. Prognosis and clinical evaluation of infection caused by Rhodococcus equi in HIV-infected patients. A multicenter study of 67 cases. Chest 2003; 123: 1970-6.

9.- McNeil M M, Brown J M. The medically important aerobic Actinomycetes: epidemiology and microbiology. Clin Microbiol Rev 1994; 7 : 357-417.

10.- Arlotti M, Zoboli G, Moscatelli G L, Magnani G, Maserati R, Borghi V, et al. Rhodococcus 
equi infection in HIV-seropositive subjects: a retrospective analysis of 24 cases. Scan J Infect Dis 1996; 28: 463-7.

11.- Farina C, Ferruzzi S, Mamprim F, Vailati F. Rhodococcus equi infection in non-HIVinfected patients: two case reports and review. Clin Microbiol Infect 1997; 3. 12-8.

12.- Meng Z H, Li Y, Huang K, Li T S, Lu H Z. Rhodococcus equi pneumonia among patients with AIDS. Clinical features and treatment. Zhonghua Yi Xue Za Zhi 2010; 90: 593-6.

13.- Mc Neil M M, Brown J M. Distribution and susceptibility of Rhodococcus equi from clinical specimens. Eur J Epidemiol 1992; 8: 437-43.

14.- Hsueh PR, Hung CC, Teng LJ, Yu MC, Chen YC, Wang HK, et al. Report of invasive
Rhodococcus equi infections in Taiwan, with an emphasis on the emergence of multidrugresistant strains. Clin Infect Dis. 1998; 27: 370-5.

15.- Guardabassi L, Christensen H, Hasman H, Dalsgaard A. Members of the genera Paenibacillus and Rhodococcus harbor genes homologous to enterococcal glycopeptide resistance genes van $\mathrm{A}$ and $v a n \mathrm{~B}$. Antimicrob Agents Chemother 2004; 48: 4915-8.

16.- Topino S, Galato V, Grillo E, Petrosillo N. Rhodococcus equi infection in HIV-infectd individuals: case reports and review of the literature. AIDS Patient Care \& STDs 2010; 24 211-22.

17.- Jacks SS, Giguère S, Nguyen A. In vitro susceptibilities of Rhodococcus equi and other common equine pathogens to azithromycin, clarithromycin and 20 other antimicrobials. Antimicrob Agents Chemother 2003; 47: 17425.

18.- Gallant J E, Ko A H. Cavitary pulmonary lesions in patients infected with human immunodeficiency virus. Clin Infect Dis 1996; 22: 671-82.

19.- de la Paz Bermúdez T, Portela Ramírez D, Jiménez Pérez N A, Dorvigny Sull M C, Kitchin Wilson M A, Capó de Paz V. Pneumonia caused by Rhodococcus equi: clinical and radiological findings in 8 AIDS patients. Rev Cubana Med Trop 2010; 62: 207-11. 